\title{
Unilateral balance training enhances neuromuscular reactions to perturbations in the trained and contralateral limb
}

\author{
Anderson Souza Castelo Oliveira ${ }^{\mathrm{a}, \mathrm{b}}$, Priscila Brito Silva ${ }^{\mathrm{a}}$, Dario Farina ${ }^{\mathrm{c}}$, \\ Uwe Gustav Kersting a,* \\ ${ }^{a}$ Center for Sensory-Motor Interaction, Department of Health Science and Technology, Aalborg University, Aalborg, Denmark \\ ${ }^{\mathrm{b}}$ The CAPES Foundation, Brazilian Education Ministry, Brasilia, Brazil \\ ${ }^{\mathrm{c}}$ Department of Neurorehabilitation Engineering, Bernstein Focus Neurotechnology Göttingen, Bernstein Center for Computational Neuroscience, University \\ Medical Center Göttingen, Georg-August University, Göttingen, Germany
}

\section{A R T I C L E I N F O}

Article history:

Received 20 August 2012

Received in revised form 17 April 2013

Accepted 18 April 2013

\section{Keywords:}

Balance training

Cross-education

Perturbations

Standing

\begin{abstract}
A B S T R A C T
The aim of this study was to investigate the effect of unilateral balance training on the reactive recovery of balance for both trained and untrained limbs. Twenty-three subjects were randomly assigned to either a control group (CG) or a training group (TG). The latter performed six weeks of balance training for the right leg. The pre- and post-training measurements were based on single leg standing posture on a moveable force platform which moved $6 \mathrm{~cm}$ anteriorly. TG subjects were tested on the trained (TR) and untrained leg (UTR), whereas CG subjects were tested on the right leg (CTR). The center of pressure trajectory length $\left(\mathrm{CP}_{\mathrm{LEN}}\right)$ and average speed $\left(\mathrm{CP}_{\mathrm{SPD}}\right)$ as well as onsets of muscular activation and time to peak $\left(\mathrm{EMG}_{\mathrm{TP}}\right.$ ) from lower limb muscles were calculated and compared by a 2-way ANOVA (three legs $\times$ two training status). Muscular onsets were reduced after training for TR $(\sim 19 \mathrm{~ms}, p<0.05)$ and UTR ( $\sim 17 \mathrm{~ms}, p<0.05)$ with no significant changes for CTR. No effects of training for $\mathrm{CP}_{\mathrm{LEN}}$ and mediallateral $\mathrm{CP}_{\mathrm{SPD}}$ were found. Furthermore, the $\mathrm{EMG}_{\mathrm{TP}}$ of UTR was predominantly greater before training $(\sim 17 \mathrm{~ms}, p<0.05)$. However, after training the $\mathrm{EMG}_{\mathrm{TP}}$ was similar among limbs. These results suggest that concomitant with improved balance recovery and neuromuscular reactions in TR, there is also a cross-education effect in UTR, which might be predominantly related to supraspinal adaptations shared between interconnected structures in the brain.
\end{abstract}

(c) 2013 Elsevier B.V. All rights reserved.

\section{Introduction}

The ability of reacting to unexpected perturbations to balance relies on the interaction between reflexes (modulated by spinal and supraspinal pathways), automatic responses and voluntary responses $[1,2]$. These mechanisms have essential implications for avoiding falls and assuring safe locomotion during daily life. Inefficient balance recovery strategies after perturbations during standing/walking are directly related to fall incidence [3]. Therefore, the use of perturbations in order to challenge balance skills and train postural control has been proposed [4-6]. Balance training (also called neuromuscular training) has been proven to reduce lower limb injury incidence and falls incidence. The use of simple devices such as wobble boards (also called ankle discs) for training purposes may reduce the injury incidence in athletes by

\footnotetext{
* Corresponding author at: Center for Sensory-Motor Interaction, Department of Health Science and Technology, Aalborg University, Fredrik Bajers Vej 7 D3, DK9220 Aalborg, Denmark. Tel.: +45 99408094; fax: +45 98154008.

E-mail address: uwek@hst.aau.dk (U.G. Kersting).
}

over 50\% [7-9]. Balance training has been effective in altering muscular reaction time (or muscle/electromyographic (EMG) onsets) to perturbations [8,10-13], improved joint positioning sense, hamstring/quadriceps ratio and joint stiffness [10,11], as well as postural sway while standing on a force platform $[8,14]$. In addition, recent investigations have shown that short-term balance training is effective in enhancing neuromuscular coordination of postural muscles, as well as neural adaptations on spinal and cortical levels [6].

Despite the fact that balance can be trained for both lower extremities, it remains to be shown whether adaptations to unilateral balance training can be transferred to the untrained limb by a cross-education effect [15]. This phenomenon has been extensively described in the literature concerning strength and resistance training $[16,17]$, in which the untrained limb also shows positive gains in strength elicited by training stimuli. Possible explanations to the cross-education effect range from peripheral to supraspinal levels (see Carrol et al. [15] for a detailed review). Recent investigations suggested that supraspinal commands play an important role for adaptations to balance training $[18,19]$, therefore, neural adaptations from unilateral balance training may 
be transferred to the untrained limb via superior levels of the CNS. However cross-education after balance training has been poorly addressed in the literature [8].

Understanding cross-education from balance training may have significant implications in neurophysiology and sports medicine. Therefore, the aim of the present study was to verify whether six weeks of unilateral balance training could enhance reactive recovery of balance during single-leg stance perturbations for the trained leg and also for the untrained leg. To achieve this aim, surface EMG and ground reaction forces were measured to determine muscle onsets and center of pressure (CoP) displacement during single leg standing perturbations. The main hypothesis was that balance training could enhance balance recovery from unexpected perturbations for the trained leg and also for the untrained leg. The optimized balance control provided by balance training could improve neuromuscular properties (muscle onsets, burst durations and magnitudes) and also be reflected in altered reactive CoP displacements, reducing its length and average speed.

\section{Methods}

\subsection{Subjects}

Twenty-three healthy men volunteered for the experiment. These subjects were randomly assigned to a training group (TG, $n=13$, age, $28 \pm 4$ years; body mass, $69 \pm 8 \mathrm{~kg}$; body height, $173 \pm 5 \mathrm{~cm}$ ) or a control group (CG, $n=10$, age, $25 \pm 3$ years; body mass, $72 \pm 8 \mathrm{~kg}$; body height, $172 \pm 3 \mathrm{~cm}$ ). All subjects were right-dominant as determined by a kicking test. Exclusion criteria included history of knee or ankle ligament injury, current lower-extremity injury, recent (within six months) low back injury, or vestibular dysfunction. All subjects provided written informed consent before participation and the procedures were approved by the ethical committee of Northern Jutland (N-20100042).

\subsection{Experimental setup}

Pre-training and post-training measurements consisted of single-leg stance perturbations to balance. Both left and right limbs were tested in a random order in TG, in one single session while for CG only the right limb was tested (Fig. 1).

The subjects were asked to stand still on a moveable force platform with their knee slightly flexed while looking straight forward at a fixed target located on a wall $4 \mathrm{~m}$ away. The free leg had to be elevated at least $5 \mathrm{~cm}$ above the platform while the hands were kept akimbo. The platform delivered forward and backward sudden perturbations to balance ( $6 \mathrm{~cm}$ length, $80 \mathrm{~ms}$ duration, average speed $75 \mathrm{~cm} / \mathrm{s}$ ). The target perturbation was the forward displacement, however, perturbations backward were included to assure unpredictability but were not analyzed. Lower limb and trunk muscle EMG and CoP displacement were recorded from $500 \mathrm{~ms}$ before the perturbation onset until $1 \mathrm{~s}$ after. A few practice trials were allowed for each direction before measurements. After habituation, 12 perturbations forward and 12 perturbations backwards were delivered in random order, with a rest interval of $10-15 \mathrm{~s}$ between them. A longer rest interval ( $2 \mathrm{~min}$ ) was provided after 12 perturbations to avoid fatigue effects.

The training protocol consisted of six weeks of balance exercises for the right limb. There were four sessions/week (24 sessions of training of 25 min duration each). The exercises were based on single leg stance performed initially on the floor and progressively increasing difficulty for balance maintenance by using foam pads, dyna discs and wobble boards (see Table 1 for exercise progression). No training stimuli were allowed for the left leg during the whole training period. Subjects of the control group were asked to maintain normal daily life activities during the 6week training program in between the two measurements.

\subsection{Kinetics}

A three-dimensional force platform (AMTI, OR6-5, Watertown, MA) mounted to a hydraulic system [20] provided perturbation stimuli and simultaneous measures of vertical $(F z)$, anterior-posterior $(F y)$ and medial-lateral $(F x)$ ground reaction forces and moments $(M x, M y$ and $M z)$. Custom-made software (MrKick II, Aalborg University, Aalborg, Denmark) was used for force recordings (1024 Hz). Using an electronic feedback circuit, the software triggered force plate movements. Ground reaction forces and moments were recorded and sampled by a kinematic tracking system (Qualisys Track Manager, Qualisys, Gothenburg, Sweden) at $256 \mathrm{~Hz}$. Signals were digitally low-pass filtered with a 4th order zero-lag Butterworth filter $(8 \mathrm{~Hz}$ cut-off). Displacement of the center of pressure (CoP) was calculated as $(x, y)=(x 0+M y / F z, y 0+M x / F z)$, where $(x 0, y 0)$ was the geometrical center of the force plate. The effects of platform movements during perturbations on the forces and moments were taken into account. A series of identical platform movements (the same delivered during the experiment) were recorded with no loads over it, in order to determine the forces and moments generated only by moving the device. Subsequently, these inertial forces and moments were subtracted from the real forces and moments used to determine the CoP.

\subsection{Electromyography}

Surface EMG signals were recorded in bipolar configuration with pairs of $\mathrm{Ag} / \mathrm{AgCl}$ electrodes (Ambu Neuroline 720 01-K/12; Ambu, Ballerup, Denmark) with $22 \mathrm{~mm}$ of center-to-center spacing. The EMG signals were amplified with a gain of 2000 (EMG-USB, LISiN; OT Bioelettronica, Turin, Italy), A/D converted (12 bit), sampled at $2048 \mathrm{~Hz}$ and band-pass filtered (second-order Butterworth, 10-500 Hz). A reference electrode was placed at the right wrist. The EMG signals of the right limb were recorded from tibialis anterior (TA), rectus femoris (RF), vastus lateralis (VL) and biceps femoris (BF) according to Hermens et al. [21]. EMG signals were synchronized to the ground reaction force by the trigger signal to the perturbation onset.

\subsection{CoP analysis}

CoP data were analyzed for each trial from the perturbation onset to $1000 \mathrm{~ms}$ after it, a period in which it is possible to regain stability after a similar perturbation
A TRAINING GROUP

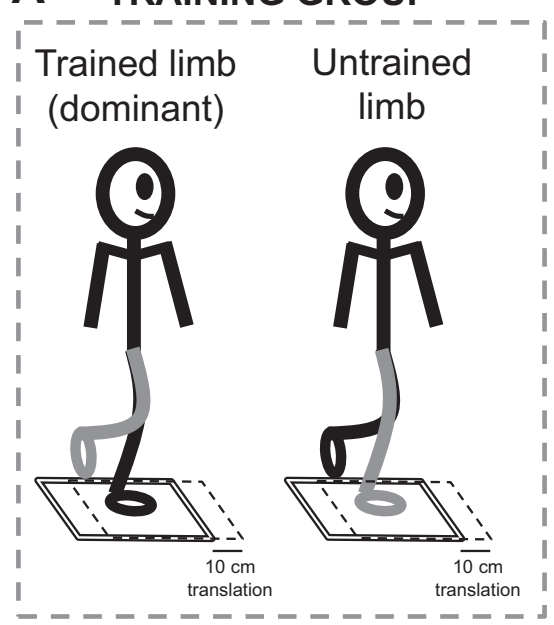

CONTROL GROUP

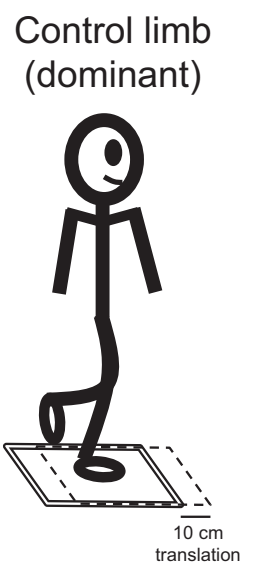

B Surface EMG

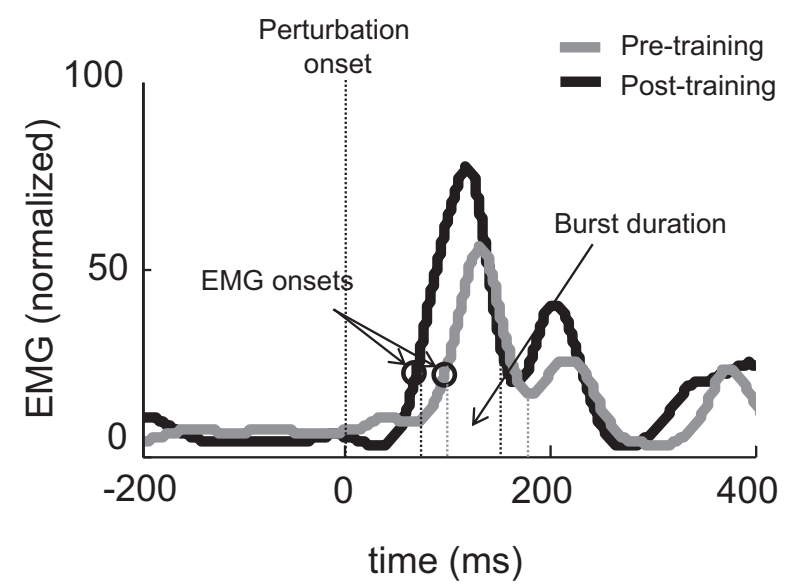

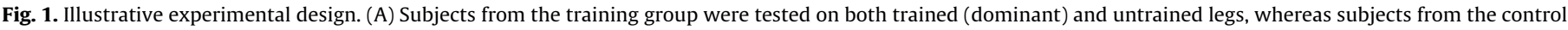

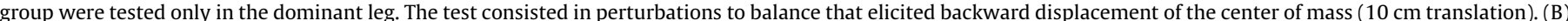

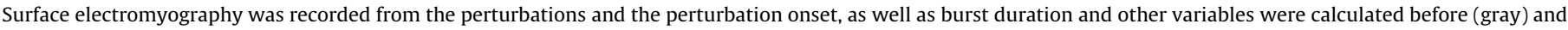
after balance training (black). 
Table 1

Balance training protocol and progression.

\begin{tabular}{|c|c|c|}
\hline Week 1 - normal floor & Week $2-3$ foam and dyna-discs & Week 4-6 wobble board \\
\hline Quiet single leg stance & Quiet single leg stance & Quiet single leg stance \\
\hline $3 \times 1 \min (\mathrm{EO})$ & $3 \times 1 \min (E O)$ & $3 \times 1 \min (E O)$ \\
\hline $3 \times 1 \min (E C)$ & $3 \times 1 \min (E C)$ & $3 \times 1 \min (E C)$ \\
\hline Single leg stance $(3 \times 1 \mathrm{~min})$ & Single leg stance $(3 \times 1 \mathrm{~min})$ & Single leg stance $(3 \times 1 \mathrm{~min})$ \\
\hline Moving head and trunk (EO) & Moving head and trunk (EO) & Moving head and trunk (EO) \\
\hline Moving head and trunk (EC) & Moving head and trunk (EC) & Ankle movements (AP and $\mathrm{ML}$ ) \\
\hline Single squats & Single squats & Single squats \\
\hline Eyes open $(2 \times 10$ reps $)$ & EO $(3 \times 10$ reps $)$ & EO $(3 \times 10$ reps $)$ \\
\hline Eyes closed ( $2 \times 10$ reps) & EC $(3 \times 10$ reps $)$ & Moving head $(3 \times 10$ reps $)$ \\
\hline Catching a ball while standing & Catching a ball while standing & Catching a ball while standing \\
\hline Low difficulty $(2 \times 90 \mathrm{~s})$ & Low difficulty $(3 \times 90 \mathrm{~s})$ & Low difficulty $(3 \times 90 \mathrm{~s})$ \\
\hline High difficulty $(2 \times 60 s)$ & High difficulty $(3 \times 60 \mathrm{~s})$ & High difficulty $(3 \times 60 \mathrm{~s})$ \\
\hline
\end{tabular}

EO: eyes open; EC: eyes closed; reps: repetition; low difficulty: the ball was catch only in front of the subject; high difficulty: the ball was catch closely or far away from the trunk, on the sides, below the knee height or above the head height.

according to Hirata et al. [22]. The following variables were analyzed to evaluate postural balance: CoP maximal excursion length $\left(\mathrm{CP}_{\mathrm{LEN}}\right)$ defined as the distance covered within $1000 \mathrm{~ms}$. CoP speed $\left(\mathrm{CP}_{\mathrm{SPD}}\right)$ defined as the average speed of the $\mathrm{CP}$ during the recovery period. $\mathrm{CP}_{\mathrm{LEN}}$ and $\mathrm{CP}_{\mathrm{SPD}}$ were calculated for both $\mathrm{AP}$ and $\mathrm{ML}$ directions.

\subsection{EMG analysis}

EMG signals were band-pass filtered (2nd order, zero-phase-lag Butterworth, 20-500 Hz), full-wave rectified, and smoothed (15 Hz low-pass, 4th order, zerophase-lag Butterworth). EMG envelopes were normalized to baseline EMG defined from a $200 \mathrm{~ms}$ interval preceding the perturbation for each individual trial.

Temporal aspects of EMG responses to the postural perturbation were assessed by the EMG onset $\left(E M G_{O N}\right)$, burst duration $\left(E G_{\text {DUR }}\right)$, burst magnitude $\left(E M G_{M A G}\right)$ and time to peak $\mathrm{EMG}\left(\mathrm{EMG}_{\mathrm{TP}}\right)$. The $\mathrm{EMG}_{\mathrm{ON}}$ for each muscle was determined as the instant in time where the amplitude surpassed two standard deviations from baseline [23]. EMG $\mathrm{DUR}_{\mathrm{D}}$ was defined as the time where EMG activity remained above the onset level within the first second after perturbations. $\mathrm{EMG}_{\mathrm{MA}}$ was determined as the integrated activity during the burst divided by the burst duration, normalized by the integral from the baseline interval. $\mathrm{EMG}_{\mathrm{TP}}$ was defined as the time from the $\mathrm{EMG}_{\mathrm{ON}}$ to the EMG maximum level of activation. In addition, EMG approximate entropy $\left(\mathrm{EMG}_{\mathrm{ENT}}\right)$ was calculated. Approximate entropy measures the (logarithmic) likelihood that runs of patterns that are close and remain close for subsequent

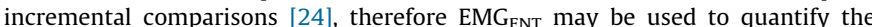
complexity or regularity of the EMG temporal series. The complexity extracted from
EMG signals is used in order to better understand neural strategies to recover balance, since highly complex EMG may suggest healthier system and/or more adaptable to environmental changes $[25,26]$, favoring performance. In this current investigation, $\mathrm{EMG}_{\mathrm{ENT}}$ was calculated from the perturbation onset to $1000 \mathrm{~ms}$ after it for each muscle separately.

\subsection{Statistical analysis}

A 2-way repeated measures analysis of variance (RM-ANOVA) was used to analyze all CoP and EMG parameters. The first factor was the tested leg with three levels (TG right leg [trained leg, TR], TG left leg [untrained leg, UTR] and CG right leg [control leg, CTR]). The second factor was time with two levels (pre-training and post-training). The Tukey LSD test was used for post hoc analysis when necessary. The data are presented as mean and standard deviation (SD). The significance level was set to $p<0.05$.

\section{Results}

\subsection{Center of pressure}

No legs $\times$ time interaction was observed for any CoP measurements $(p>0.05)$. In addition, no training effects for $\mathrm{CP}_{\mathrm{LEN}}$ on both anterior-posterior and medial-lateral components were found as
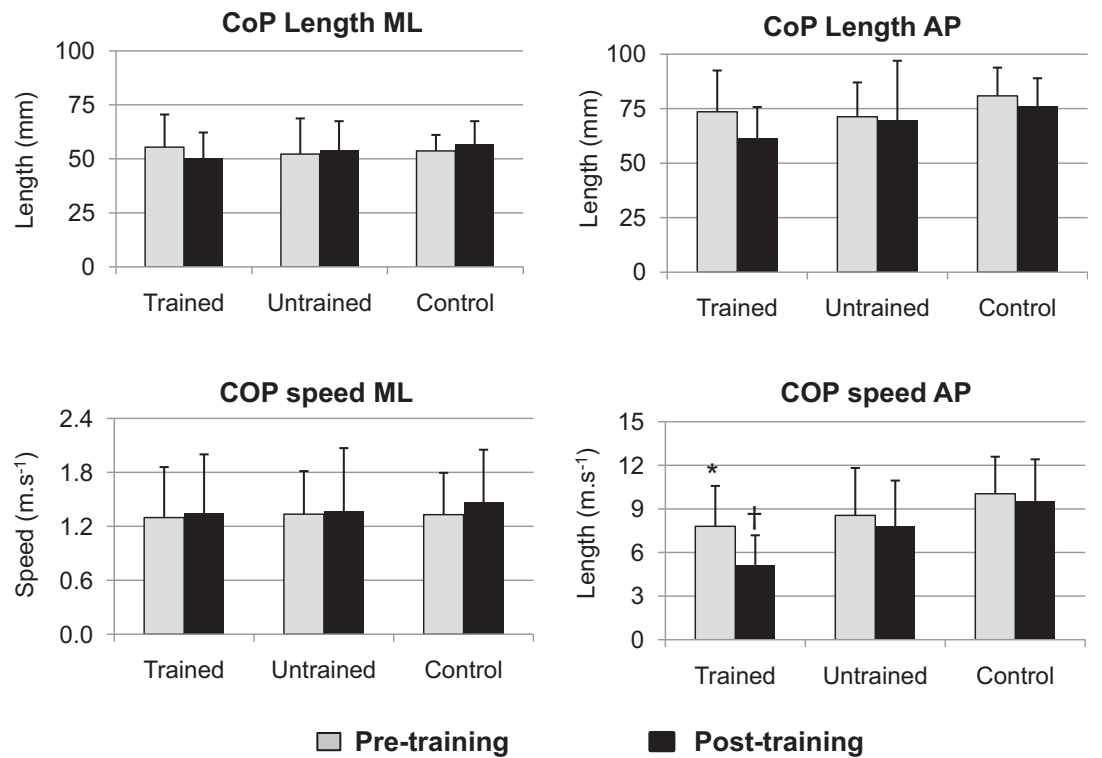

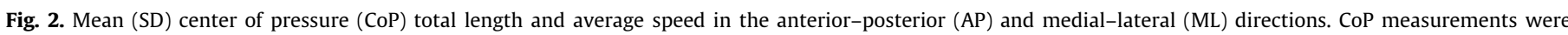

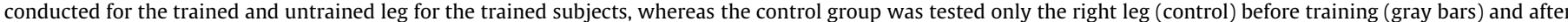

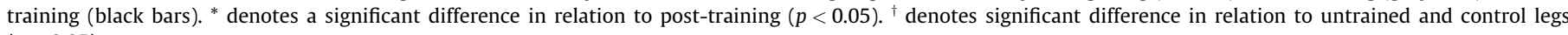
$(p<0.05)$. 
well as the medial-lateral $\mathrm{CP}_{\mathrm{SPD}}$ for TR, UTR and CTR legs (Fig. 2). On the other hand, anterior-posterior $\mathrm{CP}_{\mathrm{SPD}}$ was reduced after training for TR $(\sim 35 \%$, training effect $p<0.01)$, whereas for UTR and CTR the percental changes were $\sim 6 \%$ and $\sim 8 \%$, respectively.

\subsection{Electromyography}

No main interactions (legs $\times$ time) were observed for any of the EMG variables $(p>0.05)$, except for $\mathrm{EMG}_{\mathrm{TP}}$ and $\mathrm{EMG}_{\mathrm{ENT}}(p<0.05)$. However, activity onsets (Fig. 3, left side) were also reduced after training for all muscles in TR $(\sim 19 \mathrm{~ms}$ or $16 \%, p<0.05)$ and UTR ( $\sim 17 \mathrm{~ms}$ or $14 \%, p<0.05$ ) with no significant changes for CTR (percental change $\sim 3 \%$ ). $\mathrm{EMG}_{\text {DUR }}$ (Fig. 3, right side) was increased for all muscles in TR $(\sim 15 \%, p<0.05)$ with no changes in UTR and CTR (percental change $\sim 4 \%$ ).

EMG onsets
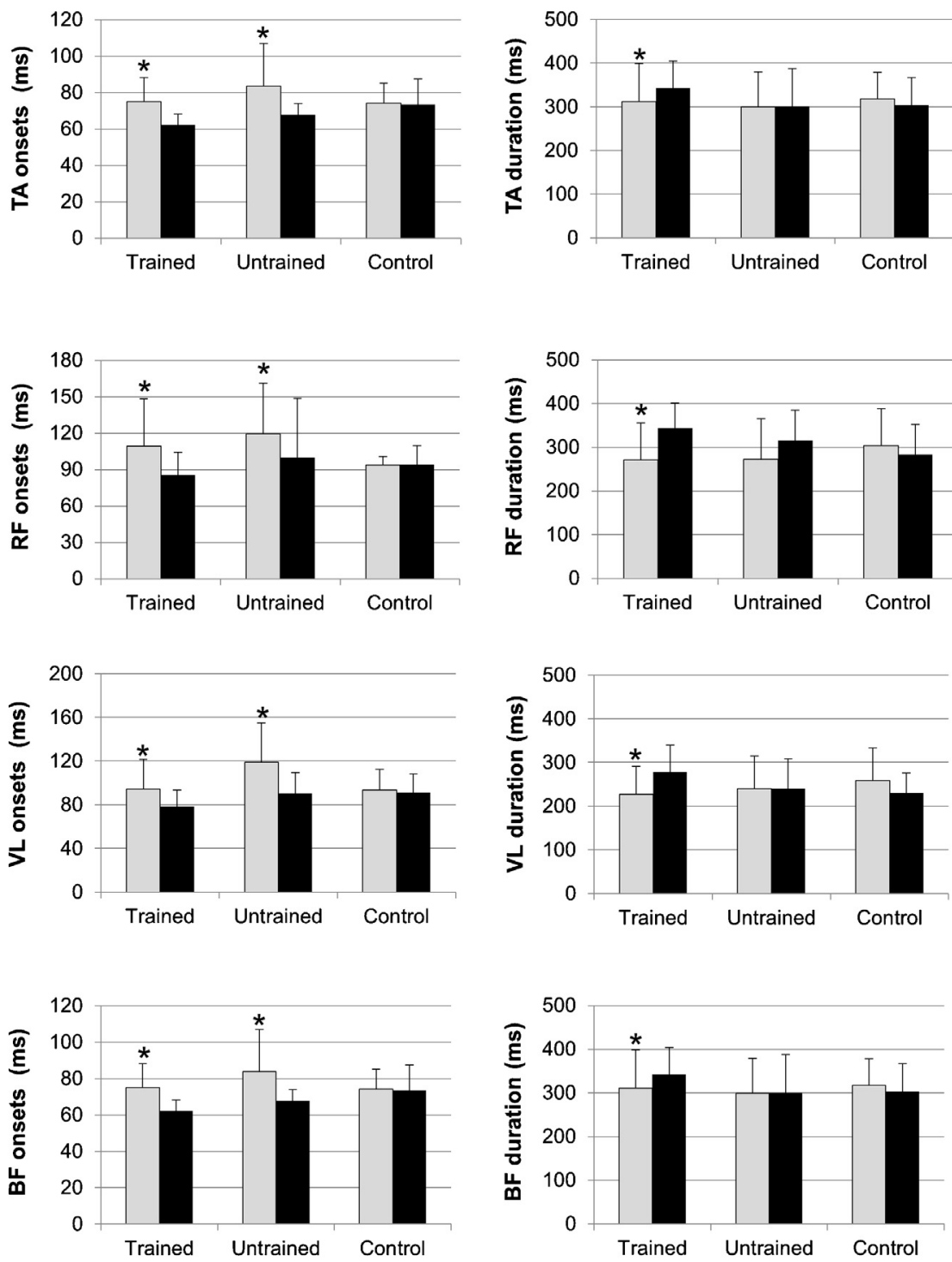

Pre-training
Burst magnitude was increased for TA and reduced for BF after training only for TR ( $p<0.05$, Table 2$)$, with no changes for UTR and CTR legs. Time to peak activity demonstrated specific changes depending on the muscle with $\mathrm{TA}$ and $\mathrm{BF}$ showing reductions ( $\sim 17 \mathrm{~ms}, p<0.01)$, and RF showing an increase $(\sim 16 \%, p<0.05)$. In addition, $\mathrm{TA}, \mathrm{RF}$ and $\mathrm{BF}$ muscles had a reduced $\mathrm{EMG}_{\mathrm{TP}}$ before training for the UTR $(p<0.05)$. After training, $\mathrm{EMG}_{\mathrm{TP}}$ was similar among

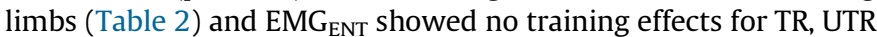
and CTR. Opposedly, UTR showed higher EMG $\mathrm{ENT}_{\mathrm{T}}$ in comparison to TR and CTR before and after training ( $p<0.05$, Table 2$)$.

\section{Discussion}

This study aimed at verifying whether unilateral balance training would improve balance recovery after forward perturbations in the
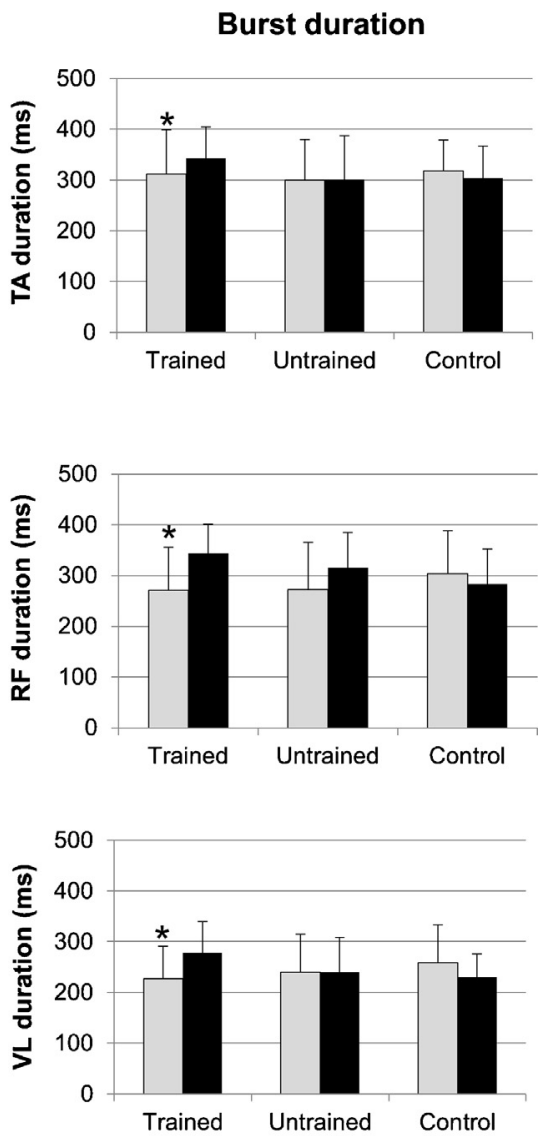

Post-training

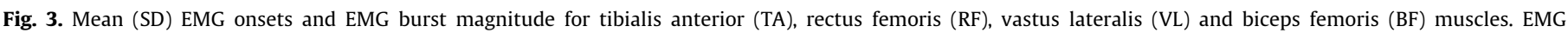

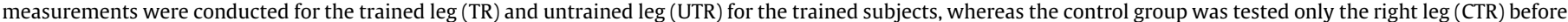

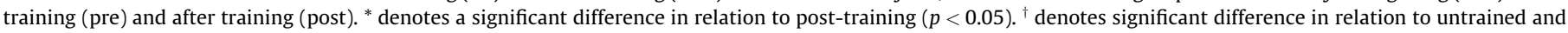
control legs $(p<0.05)$ 
Table 2

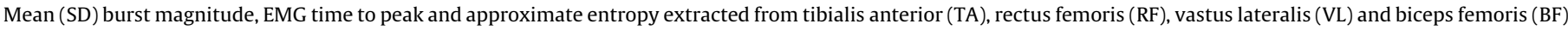
muscles.

\begin{tabular}{|c|c|c|c|c|c|c|c|c|c|}
\hline & \multicolumn{3}{|c|}{ Magnitude (\% baseline) } & \multicolumn{3}{|c|}{ Time to peak (ms) } & \multicolumn{3}{|c|}{ Entropy (a.u.) } \\
\hline & TR & UTR & CTR & $\mathrm{TR}$ & UTR & CTR & TR & UTR & CTR \\
\hline TA-pre & $993 \pm 443$ & $879.4 \pm 446$ & $831.4 \pm 352$ & $88.5 \pm 16$ & $120.3 \pm 33^{\dagger}$ & $101.9 \pm 20$ & $0.54 \pm 13$ & $0.78 \pm 28^{\dagger}$ & $0.61 \pm 12$ \\
\hline TA-post & $1421.5 \pm 404^{*}$ & $1002.8 \pm 320$ & $822 \pm 255$ & $88.7 \pm 16$ & $79.8 \pm 17^{*}$ & $89.5 \pm 18$ & $0.54 \pm 10$ & $0.86 \pm 22^{\dagger}$ & $0.59 \pm 10$ \\
\hline RF-pre & $349 \pm 128$ & $367 \pm 125$ & $349.3 \pm 135$ & $129.8 \pm 42$ & $143.1 \pm 50^{\dagger}$ & $127.6 \pm 38$ & $0.58 \pm 0.05$ & $0.93 \pm 0.3^{\dagger}$ & $0.57 \pm 0.07$ \\
\hline RF-post & $310.3 \pm 72$ & $359.1 \pm 97$ & $324.7 \pm 79$ & $154.7 \pm 46$ & $109.5 \pm 64^{*}$ & $118 \pm 32$ & $0.64 \pm 0.03$ & $0.94 \pm 0.3^{\dagger}$ & $0.59 \pm 0.07$ \\
\hline VL-pre & $280.6 \pm 95$ & 328.363 & $320.3 \pm 73$ & $94.7 \pm 33$ & $94.8 \pm 26$ & $103.6 \pm 23$ & $0.6 \pm 0.1$ & $0.93 \pm 0.2^{\dagger}$ & $0.55 \pm 0.1$ \\
\hline VL-post & $305.3 \pm 76$ & $312.7 \pm 117$ & $302.8 \pm 61$ & $113.1 \pm 47$ & $100.1 \pm 26$ & $99.2 \pm 25$ & $0.55 \pm 0.05$ & $0.90 \pm 0.2^{\dagger}$ & $0.55 \pm 0.06$ \\
\hline BF-pre & $761.9 \pm 415^{*}$ & $783.3 \pm 447$ & $569.7 \pm 328$ & $104.2 \pm 28$ & $114.3 \pm 55^{\dagger}$ & $110.1 \pm 41$ & $0.55 \pm 0.1$ & $0.77 \pm 0.2^{\dagger}$ & $0.56 \pm 0.1$ \\
\hline BF-post & $607.3 \pm 401$ & $509.9 \pm 182$ & $469.5 \pm 169$ & $91.9 \pm 34$ & $89.3 \pm 40^{*}$ & $96.1 \pm 36$ & $0.57 \pm 0.2$ & $0.66 \pm 0.1^{\dagger}$ & $0.6 \pm 0.1$ \\
\hline
\end{tabular}

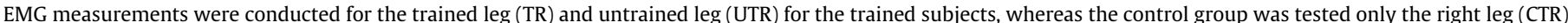
before training (pre) and after training (post).

Denotes significant difference in relation to post-training $(p<0.05)$.

Denotes significant difference in relation to untrained and control legs $(p<0.05)$

trained limb (TR), and also on the contralateral side (UTR). Our main findings were that neuromuscular responses to perturbations were enhanced after training for both legs, while the most pronounced changes were found for TR. The UTR showed faster $E \mathrm{GG}_{\mathrm{ON}}$ for all muscles and significantly reduced $\mathrm{EMG}_{\mathrm{TP}}$ for $\mathrm{TA}$ and $\mathrm{BF}$ compared to CTR. Therefore, unilateral balance training improved postural control for TR and improved initial neuromuscular responses to perturbations. These results have practical implications by demonstrating that unilateral balance training enhances recovery of balance. Moreover, these balance skills might be stimulated by a cross-education effect, leading to reduced balance loss in cases of unilateral lower limb injury. Although no interaction effects were found in the statistical analysis in some cases the relative change achieved by the training program evidently indicates adaptations for both TR and UTR legs.

Changes in $\mathrm{CoP}_{\text {LEN }}$ have been related to better postural control after balance training $[10,14]$, but no changes in this parameter were verified in the present results. However, we proposed a perturbation protocol in which the perturbation and recovery times summed were about $1 \mathrm{~s}$, much shorter than the standing task proposed in these previous studies (30 s). Moreover, the proposed balance training protocol induced reduction in the $\mathrm{CoP}_{\mathrm{SPD}}$ in the anterior-posterior direction, which indicates an enhanced ability to recover balance. These improvements on CoP variables may be related to the specific constraints imposed by different surfaces (foam, dyna discs and wobble boards). The sway patterns became less complex over time, possibly improving the efficiency of postural corrections [14].

Shorter muscular onsets and longer burst duration for TR may be interpreted as positive adaptations in terms of balance recovery allowing for rapid and prolonged muscular actions to counteract balance loss. Balance training has previously been shown to be successful in enhancing muscular onsets $[6,8,12]$, which may be related to the selection of appropriate postural reflexes, initiated by ankle proprioceptors [27]. An evident involvement of supraspinal pathways on postural responses has been demonstrated previously $[18,19,27]$, which were reflected in increased corticospinal excitability and EEG-EMG coherence [28], and increased muscular cortical representation areas [29]. Moreover, stance stability following balance training was well correlated to reduced cortical stability, but not with spinal excitability, suggesting that the most relevant adaptations to balance training are achieved at supraspinal levels [30]. In addition, strengthening of muscles, tendons, ligaments and other connective tissues are also possible [31]. Our results for TR may be the result of the sum of all these adaptations, however literature is scarce on the effect of balance training. Therefore, further investigations are needed in order to explain neurophysiologic mechanisms of adaptation following balance training.
Reduced EMG magnitude is generally found after balance training $[4,6]$, which might be related to the simplification of the motor task by learning it [6]. In the present investigation we found reduced EMG magnitude for BF muscle, but increased EMG for TA muscle in TR. BF may act knee flexor and hip extensor, this muscle is essential for hip stability, therefore a reduced BF EMG might indicate adaptations in the agonist/antagonist relationship, since there was also reduced RF EMG (not significant). The reduced EMG in these muscles would be expected, since it indicates optimized muscle recruitment by a learning effect [32]. Furthermore, the magnitude enhancement may also reflect the shorter duration of the muscle burst for TR. In the case of TA, most likely the increased EMG magnitude suggests increased responsiveness in the ankle receptors which potentialized reflex activity elicited by training. Improved initial ankle position and higher preactivation may both enhance TA responses to perturbation [33].

We have hypothesized that the positive adaptations from training could be also identified by an increased complexity in the

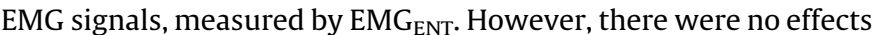
of balance training on $\mathrm{EMG}_{\mathrm{ENT}}$. It has been suggested that balance training elicits neuromuscular adaptations predominantly during early and late automatic responses (from $0 \mathrm{~ms}$ to $350 \mathrm{~ms}$ ) [5]. In order to calculate approximate entropy, the time-series must have a minimum sample size, and in this experiment the sample size was defined as 2048 ( $1 \mathrm{~s}$ from the perturbation onset). This timewindow included reflexes, automatic responses and also voluntary responses to recover balance. Therefore, the lack of adaptations in the later responses might have contributed to the lack of changes in $\mathrm{EMG}_{\mathrm{ENT}}$, further suggesting that $\mathrm{EMG}_{\mathrm{ENT}}$ might not be a good parameter to understand training adaptations in short-length time series.

Cross-education effects have been extensively studied in strength/resistance training protocols. Despite marginal contributions from peripheral/physiologic adaptation, strength training shows cross-effects by increased neural drive to the muscles, altered participation of commissural interneurons on the spinal cord, which act on the excitation/inhibition of contralateral motorneurons [15-17]. In addition, cortical adaptations mediated by interhemispheric connections via the corpus callosum might induce contralateral adaptation [15]. It is not possible to directly extrapolate adaptations from resistance training to balance training, even though, supraspinal adaptations to balance training have been reported in the literature $[5,28,30]$. In fact, a reduction in corticospinal excitability might be the main adaptation to balance training, rather than spinal adaptations, which are accompanied by improved motor performance during perturbations to balance $[6,30]$. Since interhemispheric connections might induce contralateral adaptations [15], we may suggest that supraspinal adaptation could be the primary mechanism to elicit 
cross-education following balance training. Other adaptations such as improved attention and confidence due to training might also elicit adaptations to UTR, therefore further studies must be conducted in order to clarify the underlying mechanisms related to cross-education on balance training.

In the present investigation, cross-education effects were predominantly limited to neuromuscular properties (muscular onsets, magnitudes). The slight trends to reduced $\mathrm{COP}_{\text {LEN }}$ and $\mathrm{COP}_{\mathrm{SPD}}$ may indicate limited enhancement on performance for the untrained leg. Therefore, cross-education effects occur predominantly at a neural level without requiring the execution of exercise by the limb itself for its achievement. In practical terms, the results of the present investigation suggest that neuromuscular properties of postural responses can be enhanced by a cross-education mechanism. It is not possible yet to determine whether injured patients can benefit from this cross-education effect, which could be confirmed by further studies involving injured patients.

In summary, unilateral balance training over six weeks was effective in improving neuromuscular reactions to perturbations during single-leg stance for the trained leg and to a lesser extent for the untrained leg. This suggests that balance training facilitates postural reactions when perturbations occur. The main adaptations from the trained limb about muscular onsets were also observed in the untrained limb, accompanied by reduced $\mathrm{EMG}_{\mathrm{TP}}$, which may have been acquired most likely by cortical interconnections that transfer adaptations between limbs.

\section{Acknowledgement}

The authors would like to thank the Coordenação de Aperfeiçoamento de Pessoal de Nível Superior (CAPES) for financial support to Anderson S.C. Oliveira for his PhD studies at Aalborg University (process 0293-09-1). The study was also partly sponsored by the European Project H2R ("Integrative Approach for the Emergence of Human-like Robot Locomotion", contract number 600698).

\section{Conflict of interest}

The authors declare that they have no conflict of interest.

\section{References}

[1] Duysens J, Beerepoot VP, Veltink PH, Weerdesteyn V, Smits-Engelsman BC. Proprioceptive perturbations of stability during gait. Neurophysiologie Clinique 2008;38:399-410.

[2] Rossignol S, Dubuc R, Gossard JP. Dynamic sensorimotor interactions in locomotion. Physiological Reviews 2006;86:89-154.

[3] Bieryla KA, Madigan ML. Proof of concept for perturbation-based balance training in older adults at a high risk for falls. Archives of Physical Medicine and Rehabilitation 2011:92:841-3.

[4] Horak FB, Henry SM, Shumway-Cook A. Postural perturbations: new insights for treatment of balance disorders. Physical Therapy 1997;77:517-33.

[5] Taube W, Gruber M, Gollhofer A. Spinal and supraspinal adaptations associated with balance training and their functional relevance. Acta Physiologica 2008;193:101-16.

[6] Sayenko DG, Masani K, Vette AH, Alekhina MI, Popovic MR, Nakazawa K. Effects of balance training with visual feedback during mechanically unperturbed standing on postural corrective responses. Gait and Posture 2011;35:339-44.

[7] Caraffa A, Cerulli G, Projetti M, Aisa G, Rizzo A. Prevention of anterior cruciate ligament injuries in soccer. Knee Surgery Sports Traumatology Arthroscopy 1996;4:19-21.
[8] Osborne MD, Chou LS, Laskowski ER, Smith J, Kaufman KR. The effect of ankle disk training on muscle reaction time in subjects with a history of ankle sprain. American Journal of Sports Medicine 2001;29:627-32.

[9] Wedderkopp N, Kaltoft M, Lundgaard B, Rosendahl M, Froberg K. Prevention of injuries in young female players in European team handball. A prospective intervention study. Scandinavian Journal of Medicine and Science in Sports 1999;9:41-7.

[10] Eils E, Rosenbaum D. A multi-station proprioceptive exercise program in patients with ankle instability. Medicine and Science in Sports and Exercise 2001;33:1991.

[11] Hurd WJ, Chmielewski TL, Snyder-Mackler L. Perturbation-enhanced neuromuscular training alters muscle activity in female athletes. Knee Surgery Sports Traumatology Arthroscopy 2006;14:60-9.

[12] Linford CW, Hopkins JT, Schulthies SS, Freland B, Draper DO, Hunter I. Effects of neuromuscular training on the reaction time and electromechanical delay of the peroneus longus muscle. Archives of Physical Medicine and Rehabilitation 2006;87:395-401.

[13] Sheth P, Yu B, Laskowski ER, An KN. Ankle disk training influences reaction times of selected muscles in a simulated ankle sprain. American Journal of Sports Medicine 1997;25:538-43.

[14] Strang AJ, Haworth J, Hieronymus M, Walsh M, Smart LJ. Structural changes in postural sway lend insight into effects of balance training, vision, and support surface on postural control in a healthy population. European Journal of Applied Physiology 2011;111:1485-95.

[15] Carroll TJ, Herbert RD, Munn J, Lee M, Gandevia SC. Contralateral effects of unilateral strength training: evidence and possible mechanisms. Journal of Applied Physiology 2006;101:1514-22.

[16] Dragert K, Zehr EP. Bilateral neuromuscular plasticity from unilateral training of the ankle dorsiflexors. Experimental Brain Research 2011;208:217-27.

[17] Lee M, Carroll TJ. Cross education: possible mechanisms for the contralateral effects of unilateral resistance training. Sports Medicine 2007;37:1-14.

[18] Jacobs J, Horak F. Cortical control of postural responses. Journal of Neural Transmission 2007;114:1339-48

[19] Maki B, Mcllroy W. Cognitive demands and cortical control of human balancerecovery reactions. Journal of Neural Transmission 2007;114:1279-96.

[20] van Doornik J, Sinkjaer T. Robotic platform for human gait analysis. IEEE Transactions on Biomedical Engineering 2007;54:1696-702.

[21] Hermens HJ, Freriks B, Disselhorst-Klug C, Rau G. The SENIAM Project: Surface Electromyography for Non-Invasive Assessment of Muscle. 2002.

[22] Hirata RP, Ervilha UF, Arendt-Nielsen L, Graven-Nielsen T. Experimental muscle pain challenges the postural stability during quiet stance and unexpected posture perturbation. Pain 2011;12:911-9.

[23] De Freitas PB, Knight CA, Barela JA. Postural reactions following forward platform perturbation in young, middle-age, and old adults. Journal of Electromyography and Kinesiology 2010;20:693-700.

[24] Pincus SM, Gladstone IM, Ehrenkranz RA. A regularity statistic for medical data analysis. Journal of Clinical Monitoring 1991;7:335-45.

[25] Borg FG, Laxåback G. Entropy of balance - some recent results. Journal of NeuroEngineering and Rehabilitation 2010;7:38-50.

[26] Samani A, Holtermann A, Søgaard K, Madeleine P. Advanced biofeedback from surface electromyography signals using fuzzy system. Medical and Biological Engineering and Computing 2010;48:865-73.

[27] Dietz V. Human neuronal control of automatic functional movements: interaction between central programs and afferent input. Physiological Reviews 1992;72:33-69.

[28] Perez MA, Lundbye-Jensen J, Nielsen JB. Changes in corticospinal drive to spinal motoneurones following visuo-motor skill learning in humans. Journal of Physiology 2006;573:843-55.

[29] Tyc F, Boyadjian A. Plasticity of motor cortex induced by coordination and training. Clinical Neurophysiology 2011;122:153-62.

[30] Taube W, Gruber M, Beck S, Faist M, Gollhofer A, Schubert M. Cortical and spinal adaptations induced by balance training: correlation between stance stability and corticospinal activation. Acta Physiologica 2007:189:347-58

[31] Alentorn-Geli E, Myer GD, Silvers HJ, Samitier G, Romero D, Lázaro-Haro C, et al. Prevention of non-contact anterior cruciate ligament injuries in soccer players. Part 1: mechanisms of injury and underlying risk factors. Knee Surgery Sports Traumatology Arthroscopy 2009;17:705-29.

[32] Schmid M, Bottaro A, Sozzi S, Schieppati M. Adaptation to continuous perturbation of balance: progressive reduction of postural muscle activity with invariant or increasing oscillations of the center of mass depending on perturbation frequency and vision conditions. Human Movement Science 2011;30:262-78.

[33] Weiss P, Kearney R, Hunter I. Position dependence of stretch reflex dynamics at the human ankle. Experimental Brain Research 1986;63:49-59. 